\title{
Redshift Surveys and Cosmology: A Summary of the Dunk Island Conference
}

\author{
Matthew Colless \\ Research School of Astronomy \& Astrophysics, Australian National University, \\ Mount Stromlo Observatory, Weston Creek, ACT 2611, Australia \\ colless@mso.anu.edu.au
}

Received 1999 December 1, accepted 2000 February 23

\begin{abstract}
Redshift surveys constitute one of the prime tools of observational cosmology. Imaging surveys of the whole sky are now available at a wide range of wavelengths, and provide a basis for the new generation of massive redshift surveys currently in progress. The very large datasets produced by these surveys call for new and sophisticated approaches to the analysis of large-scale structure and the galaxy population. These issues, and some preliminary results from the new redshift surveys, were discussed at the second Coral Sea Cosmology Conference, held at Dunk Island on 24-28 August 1999. This is a summary of the conference; the full conference proceedings are on the WWW at http://www.mso.anu.edu.au/DunkIsland/Proceedings.
\end{abstract}

Keywords: surveys-cosmology: observations-cosmology: large-scale structure of universegalaxies: distances and redshifts—galaxies: evolution

\section{Introduction}

The second Coral Sea Cosmology Conference, on Redshift Surveys and Cosmology, took place in the midst of a burgeoning of massive redshift surveys made possible by highly-parallel spectrographs and/or dedicated telescopes. These redshift surveys stand on the shoulders of even more massive (in terabyte terms) imaging surveys at a variety of wavelengths, which provide complete, homogeneous and complementary ways of viewing the universe and selecting samples for follow-up spectroscopy. The wealth of detail produced by these surveys is provoking more sophisticated approaches to data reduction, analysis and archiving, which offer to both improve the primary science coming out of the surveys and provide a rich resource for astronomers to mine in the coming decade.

The outline of this conference summary is as follows: Sections 2 and 3 report on the status of redshift surveys, both in progress and planned, which are mapping the local and high-redshift universe; Section 4 summarises some of the preliminary results on largescale structure derived from these and other recent surveys; Section 5 reports new results on the local galaxy population and its evolution out to high redshift; Section 6 looks to the future of redshift surveys as tools for cosmology. All references in square brackets [...] are to papers in the WWW proceedings of the conference, available at http://www.mso.anu.edu.au/ DunkIsland/Proceedings.

\section{Redshift Surveys of the Local Universe}

The Two-Degree Field facility ( $2 \mathrm{dF})$ at the AngloAustralian Telescope is a 400-fibre optical spectrograph with a $2^{\circ}$-diameter field of view. [Lewis] described how the fast robotic fibre-positioner and two focal planes of the $2 \mathrm{dF}$ make it ideally suited for massive redshift surveys. The $2 \mathrm{dF}$ Galaxy Redshift Survey has now measured redshifts for more than 50,000 galaxies, making it the largest single redshift survey to date. [Colless] gave an overview of the survey's status, reporting that as of August 1999, 227 of the 1093 survey fields have been observed, yielding redshifts for 53,192 objects, of which 50,180 are galaxies (the remainder are mostly stars plus a handful of QSOs). The redshift completeness of the survey is currently $91 \%$ and rising, as data quality and reduction methods are refined. The 2dFGRS will eventually provide redshifts and spectral information for 250,000 galaxies with $b_{J}<19.45$ (extinction-corrected) over nearly $2000 \mathrm{deg}^{2}$ (Colless 1998, 1999). Figure 1 shows the sky coverage of the 2dFGRS and a number of the other surveys mentioned here. Although the survey is at present only $20 \%$ complete, it is expected to be finished by the end of 2001.

The 2dFGRS has a companion survey in the $2 \mathrm{dF}$ QSO Redshift Survey, described by [Smith], which aims to obtain redshifts for more than 25,000 QSOs with $18.25<b_{J}<20.85$ in two strips covering $740 \mathrm{deg}^{2}$ (see Figure 1). The primary goals of the survey (Boyle et al. 1999) are to measure the primordial fluctuation power spectrum on very large scales, to obtain a geometric determination of the cosmological constant $\Lambda$, and to investigate the evolution of the QSO luminosity function and QSO clustering. Follow-ups will include studies of absorption-line and damped Lyman- $\alpha$ systems along QSO sightlines. The multicolour selection criteria for the target sample are expected to give a $93 \%$ completeness for the redshift range $0.3<z<2.2$. As of August 1999, 13,854 of the 48,078 QSO candidates have been observed, and have yielded 7026 identified QSOs. Figure 2 (top) shows both the $2 \mathrm{dF}$ galaxy and QSO surveys on a single redshift cone diagram; the density of sampling of the galaxies at low redshift is complemented by the sparser QSO sample reaching out to beyond $z=2.2$. 


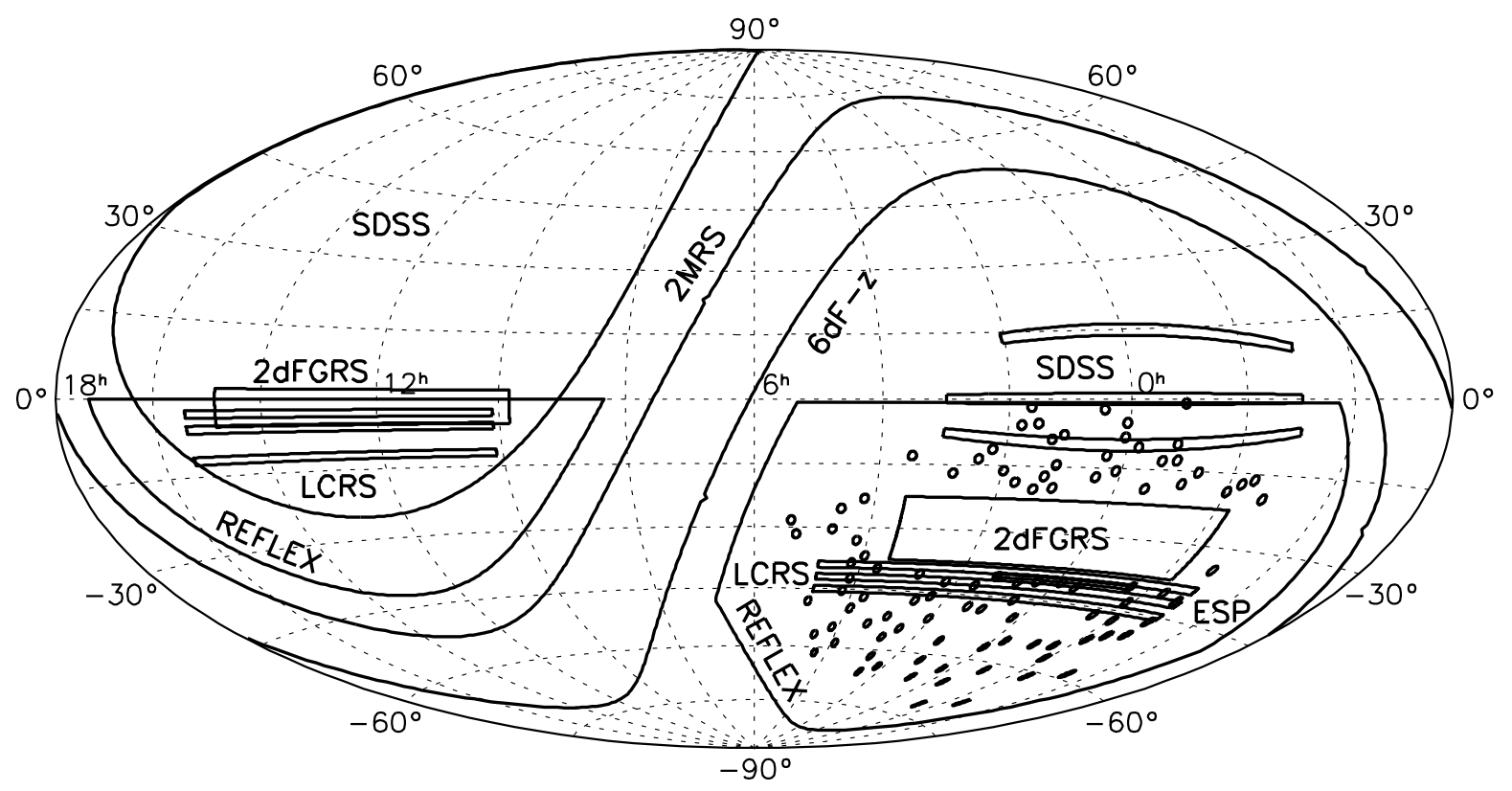

Figure 1-Areas of the sky covered by various surveys mentioned in the text.

The Sloan Digital Sky Survey (SDSS) will be one of the largest and most comprehensive astronomical datasets ever produced. Overviews of SDSS science and operations were given by [Margon] and [Szalay] (see also Margon 1999). SDSS is both a photometric and a spectroscopic survey of $\pi$ steradians of the northern sky with $b>\left|30^{\circ}\right|$. The survey uses a purpose-built $2.5 \mathrm{~m}$ telescope at Apache Point Observatory in New Mexico, supported by a $0.5 \mathrm{~m}$ telescope providing continuous monitoring of the photometric conditions. The imaging system consists of a high-efficiency drift-scan camera using $30 \mathrm{CCDs}$ to image in five passbands quasisimultaneously, while covering the sky in $3^{\circ}$-wide strips with an effective exposure time of $55 \mathrm{~s}$. This will yield images for 108 galaxies and $5 \times 10^{7}$ stars down to $r^{\prime}=$ 23.1 over a quarter of the sky. The unique SDSS photometric system $\left(u^{\prime} g^{\prime} r^{\prime} i^{\prime} z^{\prime}\right)$ was detailed by [Fukugita]. The filter set is specially designed, featuring highthroughput, nearly-disjoint passbands covering the range from 0.3 to $1.0 \mu \mathrm{m}$. The imaging survey, which achieved first light in May 1998, has already produced exciting discoveries, including the first $z=5$ QSO (Fan et al. 1999) and the coolest methane brown dwarf (Strauss et al. 1999). [Margon] listed the scientific strengths of SDSS, in ascending order of importance, as: (i) the large database; (ii) the homogeneity of the data; (iii) the well-characterised sample; (iv) discovery potential; and (v) the archival value of the imaging archive.

The volume of data to be generated by SDSS is enormous: 40 terabytes of raw data, reduced to a 'mere' 1 terabyte when processed. [Szalay] described the complex data-processing pipelines and archival database, which will generate and make available the SDSS data products, ranging from a heavily compressed all-sky map, through thumbnail images and spectra for every object, to the final photometric and redshift catalogues. Szalay also outlined the sophisticated indexing procedures that will be used to facilitate a rapid response to complex search queries.

[Loveday] described the SDSS spectroscopic survey that will be carried out in parallel with the imaging survey (Loveday \& Pier 1998). There are various spectroscopic target samples: the main galaxy sample, of 900,000 galaxies with Petrosian magnitudes brighter than $r^{\prime} \approx 18.1$; the Bright Red Galaxy sample, of 100,000 galaxies brighter than $r^{\prime} \approx 19.3$, with selection by colour and photometric redshift to give early-type galaxies with $0.25<z<0.45$; the QSO sample, of 100,000 QSO candidates down to $r^{\prime} \approx 20$, selected by colour or as FIRST or ROSAT sources; and the stellar sample, 100,000 stars of various types. In addition there will be a smaller number of serendipity sources, selected as 'interesting' by a variety of criteria. It is important to note that the redshift sample generated by this spectroscopic survey will be supplemented by photometric redshifts with a precision of $\Delta z=0.05$ for perhaps $10^{8}$ fainter galaxies, which, in conjunction with 'spectral' types based on precision colours, will be invaluable for studying the evolution of the galaxy population out to $z$ $\sim 1$. A small amount of test data has been taken with the SDSS spectrograph, a 640-fibre, manually-configured system with a $3^{\circ}$ field of view. Survey observations are expected to begin in early 2000, with the whole SDSS, both photometric and spectroscopic surveys, taking 5 years to complete.

Another view of the nearby universe is provided by the Two-Micron All-Sky Survey (2MASS), whose current status was reviewed by [Huchra]. 2MASS is surveying the whole sky in the near-infrared $J, H$ and $K_{s}$ 

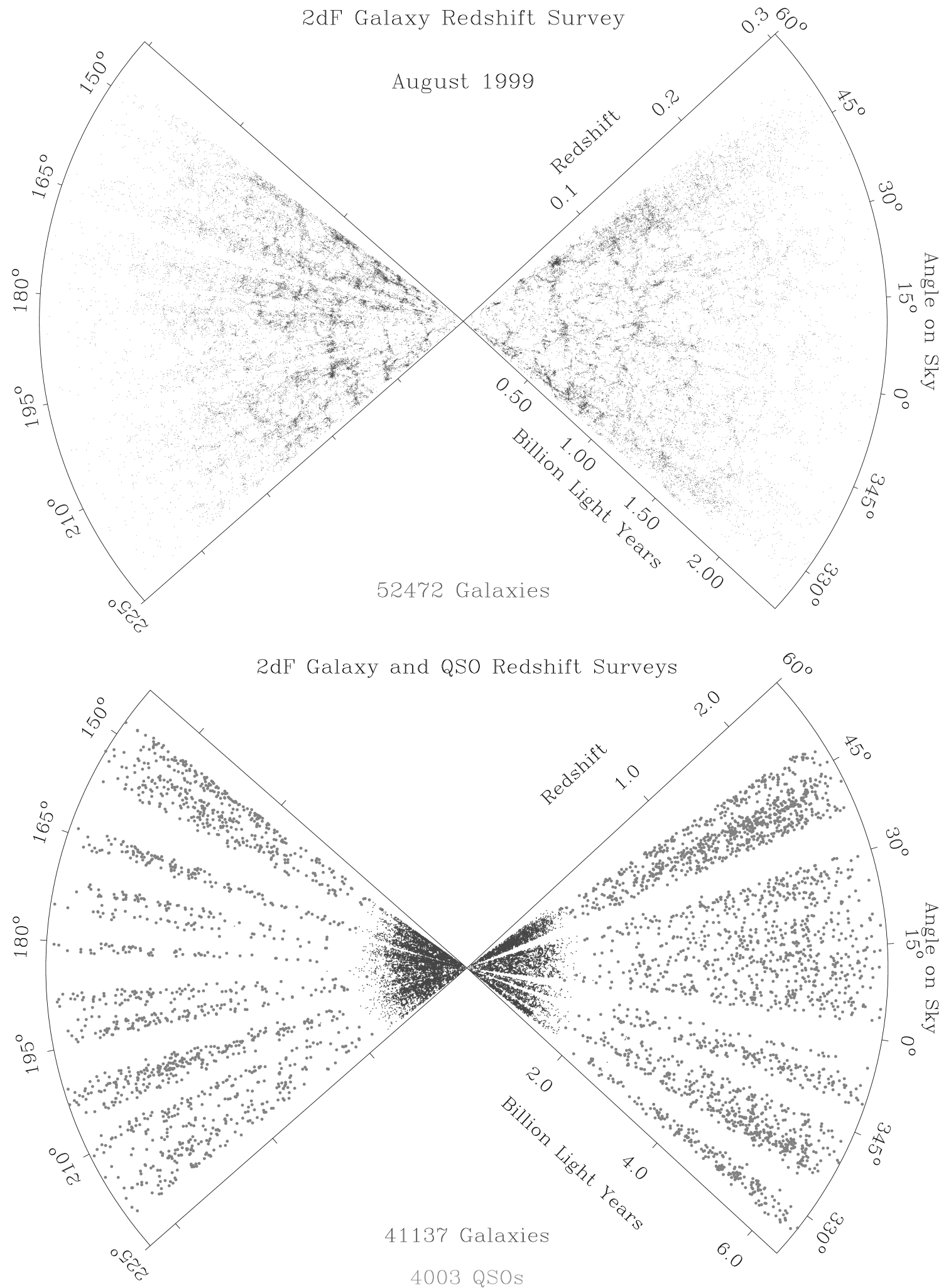

Figure 2-Redshift cone diagram showing the distribution of objects from the 2dF galaxy and QSO redshift surveys as of August 1999. The top panel shows the distribution of the galaxies alone, and the bottom panel shows the distributions of both galaxies and QSOs. 
bands, and for galaxies expects to achieve limiting magnitudes in these bands of $15.0,14.3$ and 13.5 respectively (Chester et al. 1998). The northern 2MASS survey began in 1997, and the southern survey in 1998. At present $70 \%$ of the sky has been covered by 2 MASS, and the whole survey is expected to be completed by early 2001 .

The 2MASS imaging survey will be followed up by two (coordinated) redshift surveys and two (complementary) peculiar velocity surveys. [Huchra] described the 2MASS Redshift Survey, which has the goal of measuring redshifts for 125,000 galaxies covering $90 \%$ of the sky down to $K_{s}=12.2$. This would build on existing redshift catalogues and, in the north, fill in the missing redshifts with the $1.3 \mathrm{~m}$ at FLWO used for the CfA redshift surveys. [Colless] reported plans for a southern counterpart to this survey, using the $6 \mathrm{dF}$ fibre spectrograph currently under construction for the AAO Schmidt telescope. The 6dF Galaxy Survey (Mamon 1999) has two phases, the first of which is a redshift survey of 115,000 galaxies down to $K_{S}=13, H=13.5$ or $J=14.3$ over the $17,000 \mathrm{deg}^{2}$ of the southern sky with $b$ $>\left|10^{\circ}\right|$. As well as completing the all-sky 2MASS survey to $K_{s}=12.2$ and pushing another 0.8 mag fainter, this first phase of the $6 \mathrm{dF}$ survey will provide a volumelimited sample for the second phase, a peculiar velocity survey of at least 15,000 early-type galaxies at distances less than $c z=15,000 \mathrm{~km} \mathrm{~s}^{-1}$, using the Fundamental Plane distance estimator. This in turn will complement an all-sky peculiar velocity survey of 10,000 spirals using the infrared Tully-Fisher relation proposed by [Huchra].

[Webster] reviewed the progress and some preliminary results from the HI Parkes All-Sky Survey

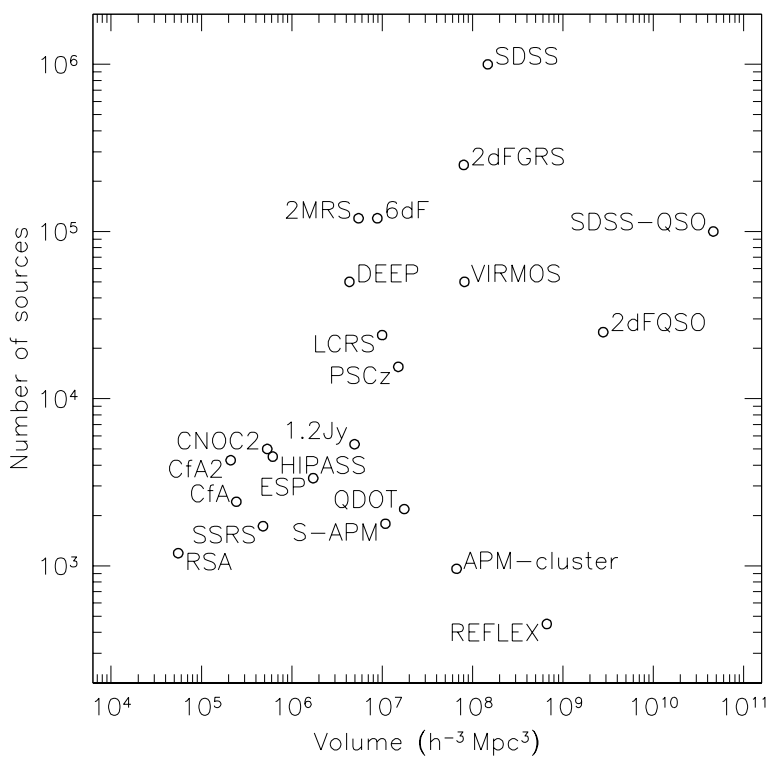

Figure 3-Comparison of the sizes and volumes of existing and planned redshift surveys. Surveys are labelled by their standard acronyms (see text).
(HIPASS; Staveley-Smith et al. 1999), which is currently using the Parkes $21 \mathrm{~cm}$ multi-beam receiver to cover the sky south of $\delta=+25^{\circ}$ out to a maximum redshift of $c z=12,700 \mathrm{~km} \mathrm{~s}^{-1}$. As of August 1999, the southern hemisphere survey is over $90 \%$ complete, and the northern extension is over $10 \%$ complete. HIPASS provides a complete $21 \mathrm{~cm}$ survey of the southern sky with 7 arcmin angular resolution down to a $3 \sigma \mathrm{HI}$ mass limit of $1.4 \times 10^{10}\left(D / 100 h^{-1} \mathrm{Mpc}\right)^{2} M_{\odot}$ (Kilborn, Webster \& Staveley-Smith 1999). When complete, HIPASS is expected to contain 5000 galaxies with a mean redshift of $3000 \mathrm{~km} \mathrm{~s}^{-1}$. The effective volume of the sample is $6 \times 10^{6} \mathrm{~h}^{-3} \mathrm{Mpc}^{3}$. HIPASS should offer a very different view of the local universe to those provided by optical and near-infrared redshift surveys, and it will be interesting to see the differences in the galaxy population and the large-scale structure, especially for low-mass objects.

Figure 3 compares the numbers of objects and the volumes encompassed by various redshift surveys.

\section{Surveys at High Redshift}

The Deep Extragalactic Evolutionary Probe (DEEP) is a two-phase survey of galaxies at redshifts out to $z \sim 1$ using the Keck Telescopes and HST. [Koo] reported results from the first phase of DEEP, now approaching completion, and outlined plans for the more ambitious second phase, which will use the DEIMOS spectrograph on Keck. The first phase (Koo 1998) utilised LRIS on Keck, in conjunction with HST imaging, to carry out a redshift survey of 1000 galaxies down to $I=24$. This survey was followed up with more detailed studies of galaxy kinematics and other physical properties for small subsamples. The second phase, using the multi-slit spectrograph DEIMOS, aims to perform a redshift survey of 50,000 galaxies to $I=23$ plus another 5000 galaxies going one magnitude deeper (Davis \& Faber 1998). The sample will be defined using photometric redshifts to select the objects with $0.7<z<1.2$. The main goals of the survey will be to study the evolution of both the galaxy population and the large-scale structure, and it is expected to run from early 2000 to late 2003.

More ambitious still is the VIRMOS survey described by [Guzzo]. This will use the optical (VIMOS) and nearinfrared (NIRMOS) multi-slit spectrographs currently being built for ESO's VLT. Using 120 nights of guaranteed time on the VLT, the survey will measure redshifts for 100,000 galaxies down to $I_{A B}=22$ over 20 $\mathrm{deg}^{2}$, and for 50,000 galaxies down to $I_{A B}=24$ over 2 $\mathrm{deg}^{2}$ (LeFevre et al. 1999). A further $\sim 1000$ redshifts will be obtained down to $I_{A B} \sim 26$ over a $1 \operatorname{arcmin}^{2}$ field, using the spectrograph's 6400-fibre integral field unit. VIMOS will see first light in mid-2000 and NIRMOS in mid-2001; the survey is expected to take 3-4 years. 


\section{Large-scale Structure}

Results on large-scale structure (LSS) were reported by a number of speakers at the conference. [Sutherland] described a determination of the power spectrum of galaxy fluctuations from the IRAS-based Point Source Catalogue redshift survey (PSCz). Using a sample of 14,500 galaxies with $60 \mu \mathrm{m}$ flux of more than $0.6 \mathrm{Jy}$ covering $84 \%$ of the sky, the PSCz team find that they are able to determine the power spectrum, $P(k)$, for wavenumbers down to $0.03 h \mathrm{Mpc}^{-1}$ (i.e. scales up to $200 h^{-1} \mathrm{Mpc}$ ). The results (Sutherland et al. 1999; Tadros et al. 1999) are consistent with the earlier QDOT and 1.2 Jy surveys, although $P(k)$ is significantly better determined. For plausible values of the small-scale velocity dispersion, $P(k)$ is well-fit by a CDM-like model with shape parameter $\Gamma \approx 0.25$ and normalisation $\sigma_{8} \approx 0.75$ (although assuming a larger dispersion permits a model with $\Gamma \approx 0.5$ to be marginally acceptable).

[Rowan-Robinson] reported an estimate of $\beta=$ $\Omega^{0.6} / b$ derived from the comparison of the Local Group (LG) motion with respect to the CMB and the motion predicted from a model based on clusters and voids identified in the PSCz and the literature (see also Schmoldt et al. 1999a, 1999b). The predicted LG dipole has nearly converged by $z=0.1$ (with most of the LG motion generatedwithin $200 \mathrm{~h}^{-1} \mathrm{Mpc}$ ); linear theory requires $\beta \approx 0.7$ to fit the observed amplitude of the $\mathrm{LG}$ motion.

[Guzzo] summarised the results of the ESO Slice Project (ESP; Vettolani et al. 1997) redshift survey. This precursor to surveys like the 2dFGRS used photographic sky survey plates to select a sample of galaxies down to $b_{J}=19.4$ in a single $1^{\circ} \times 35^{\circ}$ strip. Redshifts were obtained for 3342 galaxies ( $85 \%$ complete; Vettolani et al. 1998). Perhaps the most significant LSS result to emerge from ESP is that there is good evidence for a local under-density of nearly a factor of 2 extending out to $250 h^{-1} \mathrm{Mpc}$ (Zucca et al. 1997). This is consistent with the normalisation difference between the luminosity functions derived from surveys at this depth (ESP itself and the Autofib survey; Ellis et al. 1996) and those derived from shallower surveys such as APM-Stromlo (Loveday et al. 1992). It also 'explains' the origin of the steep number counts at bright magnitudes (Maddox et al. 1990), although it begs the question of the completeness of the galaxy catalogues derived from photographic surveys at bright magnitudes. The local void implied by this result would appear to cover much of the south Galactic cap, as evidenced by the north/south differences in galaxy density found in the PSCz, LCRS and CfA2 surveys. Is this void, with an amplitude $\delta \rho / \rho \approx 0.5$ compatible with statistical measures of clustering? The ESP redshift-space correlation function is in good agreement with other determinations on scales between 1 and $50 h^{-1} \mathrm{Mpc}$, though a little lower on the smaller scales. The power spectrum is likewise consistent with other determinations at wavenumbers $k>0.1 \mathrm{~h} \mathrm{Mpc}^{-1}$. However, the small volume covered by the survey, and its strip geometry, prevent a reliable determination of $P(k)$ at wavenumbers below about $0.1 h \mathrm{Mpc}^{-1}$ (i.e. scales above $60 \mathrm{~h}^{-1} \mathrm{Mpc}$ ).

An efficient approach to surveying larger volumes, and so measuring $P(k)$ at smaller $k$, is to use redshift surveys of clusters, since clusters are about five times more strongly clustered than galaxies. Results from two such surveys were summarised at the meeting. [Boehringer] and [Guzzo] reported early results from the REFLEX survey, while [Tadros] summarised the results from the first stage of the APM cluster survey.

[Boehringer] noted the advantages of an X-ray selected cluster sample as being the close correlation between $L_{X}$ and mass, and the minimisation of projection effects. He described the detection of clusters in the ROSAT All Sky Survey for the REFLEX (southern) and NORAS (northern) samples (Guzzo et al. 1999). Together these samples comprise over 900 clusters covering the whole sky at latitudes $b>\left|20^{\circ}\right|$ down to a flux limit of $F_{X}=3 \times 10^{-12} \mathrm{erg} \mathrm{s} \mathrm{cm}{ }^{2}$. The preliminary power spectrum derived from the REFLEX survey using a subset of 188 clusters in a $400 h^{-1}$ Mpc comoving cube (Schuecker et al. 1998) appears to show a significant turnover in $P(k)$ at $k \approx 0.04-0.05 h \mathrm{Mpc}^{-1}$, corresponding to $125-160 \mathrm{~h}^{-1} \mathrm{Mpc}$. [Guzzo] presented very preliminary results from a larger sample of clusters in a $1000 h^{-1} \mathrm{Mpc}$ comoving cube that confirm the turnover in $P(k)$, but the newer results also hint at a possible second peak in $P(k)$ at $k \sim 0.01$, although an $\Omega_{\Lambda}$ $=0.7$ flat $\mathrm{CDM}$ model remains consistent within the (large) uncertainties.

[Tadros] described a similar survey, the APM Cluster Redshift Survey (Tadros, Efstathiou \& Dalton 1998). The first phase of this survey consisted of 364 clusters drawn from the APM cluster catalogue of 960 clusters covering $4500 \mathrm{deg}^{2}$. The APM clusters provide a cleanly-selected sample that is largely unaffected by the inhomogeneities and projection effects afflicting the Abell catalogue. The redshift-space cluster-cluster correlation function $\xi_{c c}(s)$ is well represented by a power law with index $\gamma \sim 2$ and a correlation length of $14 h^{-1}$ Mpc (smaller than that of the Abell catalogue). The LSS as measured from clusters provides a clean comparison with cosmological models, since clusters are readily identified from the dark matter halos without the confusion of a bias parameter. The APM $\xi_{c c}(s)$ is consistent with $\Lambda \mathrm{CDM}$ and MDM models, but has more clustering than is predicted by standard CDM.

An estimate of $\beta=\Omega^{0.6} / b$ can be obtained by comparing the real-space and redshift-space cluster-galaxy cross-correlation functions $\xi_{c g}$. The real-space $\xi_{c g}(r)$ was derived from the cluster survey and the APM galaxy catalogue by inverting the angular cluster-galaxy cross-correlation function. The redshift-space $\xi_{c g}(\sigma, \pi)$, as a function of separation in the planeof the sky $(\sigma)$ and along the line of sight $(\pi)$, was derived from the cluster survey and the Stromlo-APM redshift survey. These two $\xi_{c g}$ values can be related using a model incorporating 
nonlinear infall and the velocity dispersion of galaxies around clusters. [Tadros] showed that the best-fit model yields an estimate of $\beta \approx 0.4$, with a $95 \%$ upper confidence limit of $\beta<0.7$. The power spectrum from the APM cluster survey shows a turnover at $k \approx 0.03 h$ $\mathrm{Mpc}^{-1}$, but the median depth of the survey $\left(270 \mathrm{~h}^{-1}\right.$ $\mathrm{Mpc})$ is only just adequate to measure $P(k)$ on this 200 $h^{-1} \mathrm{Mpc}$ scale. To improve the significance of this detection and reduce the systematic errors, the second phase of the APM cluster survey, now under way, will obtain redshifts for the remaining clusters in the APM cluster catalogue, bringing the sample up to 960 clusters. This cluster survey, which will take 2-3 years to complete, will be particularly useful since it overlaps with the 2dF Galaxy Redshift Survey.

The faint galaxy correlation function at large angular scales was discussed by [Brown], who has used digitally-stacked Schmidt plates to achieve an approximate limit of $B_{J}=23.5$. This gives 700,000 galaxies in each of two $40 \mathrm{deg}^{2}$ fields. The median redshift at this depth is $z \sim 0.4$. The correlation function he derives is well-fit as a power law, $\omega(\theta) \propto \theta^{1-\gamma}$ with $\gamma=1.7$, over the range 0.05-10 $h^{-1} \mathrm{Mpc}$. The amplitude of the correlation function declines as $(1+z)^{-(3+\epsilon)}$ with $\epsilon \approx 0$, corresponding to fixed clustering in physical coordinates. However, these results hide the different clustering properties of the red and blue galaxies, which are respectively fitted by models with $\gamma=1.8, \epsilon=-1.3$ and scale length $r_{0}=8.6 h^{-1} \mathrm{Mpc}$, and $\gamma=1.6, \epsilon=-1.5$ and $r_{0}=3.5 h^{-1} \mathrm{Mpc}$. The clustering amplitude of red galaxies is thus about five times higher than that of the blue galaxies. The lack of any significant strengthening of the clustering amplitude of blue galaxies out to $z \sim 0.4$ suggests that the increase in the population of blue galaxies in clusters with redshift (the Butcher-Oemler effect) is simply related to the overall increase in the faint blue galaxy population with redshift.

The LSS goals of the 2dFGRS, and some preliminary results from the survey, were described by [Dalton]. The main LSS goals are: (i) the determination of $P(k)$ on large scales (>100 $h^{-1} \mathrm{Mpc}$ ); (ii) the topology of the 3D distribution; (iii) tests of the Gaussian nature of the density field (on large scales) and biasing of the galaxies with respect to the mass (on small scales) from higher-order statistics; and (iv) estimation of the mass density $\Omega$ and the bias parameter(s) $b$ from the redshiftspace distortions. Dalton discussed the tiling and fibreassignment algorithms used in the survey, and showed that the restrictions on minimum fibre separations mean that the survey is significantly biased against close pairs with separations less than 2 arcmin (corresponding to $150 h^{-1} \mathrm{kpc}$ at the median depth of the survey, $z=0.1$ ). This effect, and the variation in redshift completeness with apparent magnitude, are taken into account when estimating or simulating the LSS statistics derived from the survey.

[Dalton] showed preliminary determinations of the redshift-space correlation function $\xi(\sigma, \pi)$ for the 2dFGRS. There is very good agreement with the results obtained from the Las Campanas Redshift Survey (LCRS). The distortions in $\xi(\sigma, \pi)$ shown in Figure 4 are roughly consistent with a model with $\beta=\Omega^{0.6} / b \approx 0.5$ and smallscale velocity dispersion $\sigma \approx 400 \mathrm{~km} \mathrm{~s}^{-1}$. A similar value for $\beta$ emerges from the quadrupole-to-monopole ratio of the redshift-space distortions on large scales. As a consistency check on the results, the projected correlation function $\Xi(r)$, derived by integrating over $\xi(\sigma, \pi)$, has been compared to that obtained by Baugh \& Efstathiou (1993) from a deprojection of the angular correlation function of the parent APM galaxy cata-
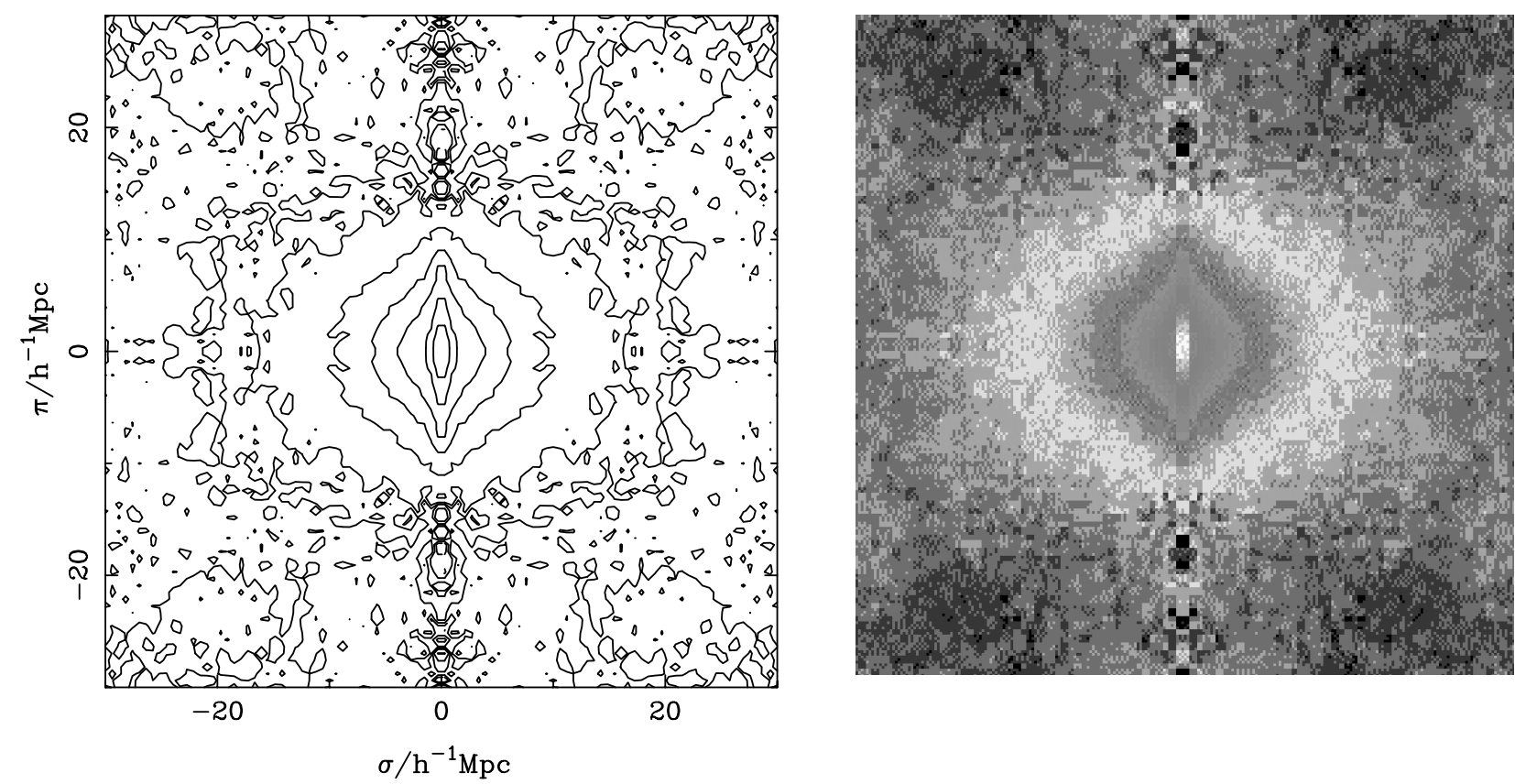

Figure 4-The 2dFGRS redshift-space correlation function, $\xi(\sigma, \pi)$, shown as both a contour plot (left) and a false-colour image (right). Note the stretching/flattening along the line of sight $(\pi)$ for small/large separations in the plane of the sky $(\sigma)$. 

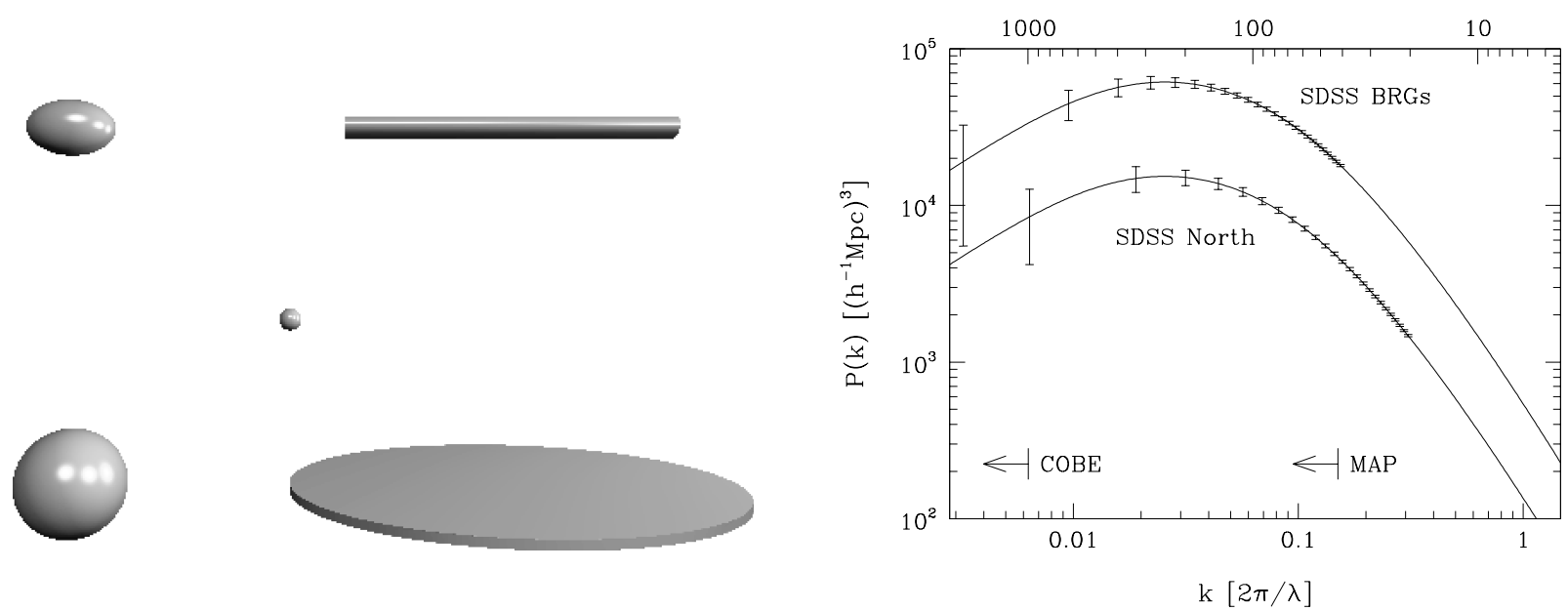

Figure 5-(left) A comparison of the window functions for various surveys, showing the significant improvement in resolution of the power spectrum that is achieved as the surveys increase in both sample size and sky coverage, from the BEKS pencil-beam survey (lower right), through the LCRS slice survey (upper right) and the CfA2 survey (upper left), to the QDOT sparse all-sky survey (lower left), and finally the 2dFGRS and SDSS surveys (centre) (from the SDSS Black Book). (right) The prediction for the recovered power spectrum and estimated errors from the SDSS main northern galaxy sample and from the Bright Red Galaxy sample (Loveday \& Pier 1998). The scales covered by the COBE and MAP cosmic microwave background probes are also indicated.

logue. There is excellent agreement on scales up to 30 $h^{-1} \mathrm{Mpc}$, while on larger scales the cosmic variance dominates the uncertainties in the as-yet-incomplete 2dFGRS.

In a similar vein, [Szalay] showed the results of simulations indicating the precision that the Sloan survey will achieve in recovering cosmological parameters from LSS statistics. SDSS should be able to measure the power spectrum normalisation $\sigma_{8}$ to a precision of $5 \%$, the power spectrum shape parameter $\Gamma$ to $20 \%$, and the redshift-distortion parameter $\beta$ (which involves both the mass density $\Omega$ and the bias parameter $b, \beta=\Omega^{0.6} / b$ ) to $35 \%$. The precision with which $P(k)$ will be determined is illustrated in Figure 5, which shows both the effective window functions of various surveys and the simulated recovery of $P(k)$ from the Sloan survey, with estimated errors.

On larger scales of both space and time, [Croom] reported preliminary LSS results for the $2 \mathrm{dF}$ QSO Redshift Survey. The LSS goals of the QSO survey are: (i) determining the QSO $P(k)$ out to scales of $1000 h^{-1}$ $\mathrm{Mpc}$; (ii) measuring the cosmological constant $\Lambda$ from geometrical (as opposed to dynamical) distortions of clustering in redshift space; and (iii) tracing the evolution of QSO clustering out to $z \sim 3$ to constrain $\Omega$ and the QSO bias parameter. As with the galaxy survey, corrections are needed for the partial coverage of overlapping fields, the deficit of close pairs and Galactic extinction. Preliminary determinations of the $0.3<z<$ 2.2 QSO correlation function using 2765 QSOs give $\gamma \approx$ 1.4 and scaling lengths $r_{0} \approx 3 h^{-1} \mathrm{Mpc}$ and $r_{0} \approx 5 h^{-1}$ Mpc for models with $\left(\Omega_{M}=1, \Omega_{\Lambda}=0\right)$ and $\left(\Omega_{M}=0.3\right.$, $\Omega_{\Lambda}=0.7$ ) respectively, with the clustering appearing to be constant in comoving coordinates over this range. With the full QSO survey, it should be possible to measure $\gamma$ to $3 \%$ and $r_{0}$ to $5 \%$ on small scales, and on large scales to measure $P(k)$ on scales $k>0.01 h \mathrm{Mpc}^{-1}$ (i.e. $<600 h^{-1} \mathrm{Mpc}$ ).

Other cosmological parameters were addressed by Mould and Peterson. [Mould] summarised the results from the HST Key Project to measure the Hubble constant, $H_{0}$ (Mould et al. 2000). This work essentially consists of using Cepheid distances to calibrate a wide variety of distance estimators, including type Ia supernovae, the Tully-Fisher relation for spiral galaxies, and the Fundamental Plane and surface brightness fluctuations for bulge-dominated galaxies. The available data from all these estimators yield consistent values for $H_{0}$, and the combined best estimate, after correcting for the chemical composition dependence of the Cepheid period-luminosity relation, is $H_{0}=(68 \pm 6) \mathrm{km} \mathrm{s}^{-1} \mathrm{Mpc}^{-1}$ (including random and systematic errors), for an assumed LMC distance of 50 $\pm 3 \mathrm{kpc}$. [Rowan-Robinson] also gave an estimate of the Hubble constant using a similar compilation of methods based on the Key Project Cepheid distances, but with the addition of corrections for peculiar velocities based on the PSCz flow model. He finds $H_{0}=(65 \pm 2) \mathrm{km} \mathrm{s}^{-1} \mathrm{Mpc}^{-1}$ (random error only).

[Mould] also considered the possibility that we inhabit a large local void with $-0.5<\delta n / n<-0.2$, as suggested by [Guzzo] from the results of the ESP survey, and as is consistent with the preliminary results from the 2dFGRS. Since $\delta H_{0} / H_{0}=\frac{1}{3} \beta \delta n / n$, this would imply (for $\beta=\Omega^{0.6} / b \approx 0.5$ ) that $0.92<H_{0}$ global $/ H_{0}$ local $<0.97$. Mould concluded that while further weak constraints on $\delta n / n$ can be derived from galaxy number counts, the issue should be settled by the 2dFGRS and SDSS redshift surveys, which together sample the density field in both hemispheres.

[Peterson] addressed the question of whether a cosmological constant is demanded by the galaxy num- 
ber counts. He finds that the surface density of faint galaxies derived from the optical and near-infrared number counts is too high to be compatible with a $\Omega_{M}=$ 1 cosmology, and are much better fitted by a lowdensity flat universe with $\Omega_{\Lambda} \approx 0.8$. Although this claim depends on the assumed evolutionary history of the galaxies, Peterson argued that models that reproduce the number counts by invoking merger-driven evolution are inconsistent with the low measured amplitude of the angular correlation function for faint galaxies.

\section{Galaxy Population and Evolution}

The new generation of large redshift surveys promise to reveal the properties of the galaxy population in extraordinary detail. Some of this potential was revealed by various preliminary results emerging from the $2 \mathrm{dFGRS}$. [Colless] summarised some of the recent work by members of the survey team on the local luminosity function (LF). The overall LF shows clear signs of being more complex than the standard Schechter form, with a steepening of the faint end for $M_{b_{J}}>-16$. Using a principal component analysis (PCA) to classify the galaxies into five spectral types (Folkes et al. 1999), it is clear that this is the result of a general steepening of the faint end for later-type galaxies, which dominate the population at lower luminosities.

However, this type of analysis does not help explain the large variation between different determinations of the local LF, not only in the faint end but also in the normalisation. A more general approach is required, which will account for surface brightness selection effects. [Colless] reported a preliminary determination (Cross et al., in preparation) of the bivariate brightness distribution (BBD), the joint distribution over absolute magnitude and surface brightness. This shows that the 2dFGRS is not severely affected by its selection limit at low surface brightness, as the galaxy number distribution falls off sharply before that limit is reached. The $\mathrm{BBD}$ is strongly suggestive of the existence of separate dwarf and giant populations, the former with a LF that is flat or declining at the faint end, and the latter with a steeply increasing faint end.

More detailed analyses of the galaxy population can be carried out using various quantities derived from the spectra: [Colless] showed that measurement of the $\mathrm{H} \alpha$ equivalent width can be used to map the relative distributions of the star-forming and quiescent galaxies in the 2dFGRS (Lewis et al., in preparation), while [Deeley] used the Balmer-line and [OII] equivalent widths to identify post-starburst $\mathrm{E}+\mathrm{A}$ galaxies, finding an incidence of $0.25 \%$.

A very powerful application of the redshift surveys is in the identification of sources detected in sky surveys at other wavelengths. [Cannon] reported on a program to identify the NVSS and SUMSS radio galaxies in the 2 dFGRS (Sadler et al. 1999). About $5 \%$ of NVSS sources are found in the $2 \mathrm{dFGRS}$, and about $1.5 \%$ of 2dFGRS sources are in NVSS; in the full redshift survey there should be about 4000 radio galaxies. The 700 sources identified so far, with roughly equal numbers of star-forming galaxies, optical AGN and radio AGN, already make up the largest homogeneous sample of optical spectra for radio galaxies. The first result emerging from this sample is a much-improved determination of the radio luminosity function, which has a double-humped appearance. By using the PCA spectral types, [Cannon] showed that this structure is due to different luminosity functions for the AGN (which dominate at high luminosity) and the star-forming galaxies (which have a steeper slope and dominate at lower luminosities).

[Jackson] described the potential for future work combining radio surveys such as NVSS, SUMSS and FIRST with large redshift surveys such as the $2 \mathrm{dF}$ galaxy and QSO surveys, SDSS and the 6dF survey. Understanding the properties of radio galaxy populations is very much hindered by the lack of large, homogeneous redshift samples. Cross-identification with the 2dFGRS allows the definition and detailed studies of the local radio galaxy populations: the luminosity functions (see above), environmental effects, clustering and low-redshift evolution. Cross-identification with the $2 \mathrm{dF}$ QSO survey allows similar studies of radio-loud QSOs. Limitations arise from the joint radio/optical selection criteria; nonetheless such studies promise to greatly improve our understanding of radio galaxies.

[Boyle] showed the results obtained on QSO evolution using a homogeneous sample of 3265 QSOs from the $2 \mathrm{dF}$ QSO Redshift Survey. He finds that the luminosity function is fitted by a double power law (i.e. it has a break), and that the change in the LF with redshift is consistent with a $\Omega_{M} \approx 0.3, \Omega_{\Lambda} \approx 0.7$ universe and pure luminosity evolution with the approximate form $L^{*}(z) / L^{*}(0) \propto \operatorname{dex}\left(1.4 z-0.27 z^{2}\right)$ out to $z=2.3$. He noted that the SDSS QSO survey, which is not limited by UVX selection, may be able to see whether this evolution turns around for redshifts between 2.3 and 3 , with decreasing numbers of QSOs at fixed luminosity. He also reported results from HST and ground-based imaging of a sample of 76 low-redshift, X-ray selected AGN (Schade et al., in preparation). Host galaxies with $-23<M_{\text {host }}<-17$ were detected in all cases, with $55 \%$ of AGN residing in bulge-dominated galaxies. A weak correlation is found between the AGN and host luminosities. Interestingly, $10 \%$ of AGN hosts do not show a point source (in agreement with HST studies of fainter AGN). The luminosities, sizes and morphologies of the AGN hosts are completely consistent with the 'normal' galaxy population, except that AGN preferentially reside in bulge-dominated systems.

[Giavalisco] presented some recent results from surveys of Lyman-break galaxies (LBGs) at $z \sim 3$ and $z$ $\sim 4$ (Giavalisco et al. 1998; Steidel et al. 1999). The colour-selection of LBGs is a highly efficient method for generating samples of star-forming galaxies at high 
redshift. Follow-up spectroscopic observations have now provided redshifts for more than 750 galaxies with $z=2-5$. The selection biases are believed to be wellunderstood, so that it is possible to compute the farultraviolet luminosity function (LF) for the LBGs. Combining ground-based and Hubble Deep Field samples, the $z \sim 3 \mathrm{LF}$ (measured in the $R$ band, which corresponds to $\sim 1700 \AA$ in the rest-frame) is found to be well-represented by a Schechter function with a steep faint end $(\alpha \approx-1.6)$, although the slope does depend on the incompleteness correction that is applied. With assumptions about the LBG spectral energy distribution, and using the Calzetti (1997) reddening law, it is possible to estimate the extinction by dust in the LBGs. The median extinction at rest-frame $1500 \AA$ is found to be $1.7 \mathrm{mag}$, and integrated over the whole population the UV extinction is about a factor of 7. The smaller spectroscopic sample of LBGs selected at $z \sim 4$ does not show any significant change from the $z \sim 3$ population: the LFs of both samples are consistent in shape and normalisation. The LBGs are very strongly clustered, with comoving correlation function amplitude at least as great as that of $z \sim 0$ galaxies. Given the weaker mass clustering at early epochs, this implies much stronger biasing. However, this picture is consistent with the predictions of simple analytic models (e.g. Mo, Mau \& White 1999) for the evolution of galaxy clustering based on the measured power spectrum for low-redshift galaxies.

Francis and Illingworth reported studies of highredshift galaxy clusters. [Illingworth] showed HST results relating to the nature of galaxies in rich environments at $z \sim 0.3-1$. The mild evolution in the Fundamental Plane for the E and S0 galaxies in the cluster CL1358+62, relative to the nearby Coma cluster, implies that these galaxies are a mature, homogeneous population whose stars formed at redshifts $z>1$ (Kelson et al. 2000). The absorption line strengths are also consistent with old, single-burst populations in which metallicity increases with velocity dispersion. For earlytype spirals, however, there is a positive correlation between luminosity-weighted age and velocity dispersion. In contrast to this evidence for a high redshift of star formation in early-type galaxies, [Illingworth] also reported results from a morphological study of the cluster MS1054-03 at $z=0.83$ that show a high merger rate in this luminous $\mathrm{X}$-ray cluster. If this rate is typical for clusters at this redshift, it implies that up to $50 \%$ of the massive ellipticals in clusters may be formed in mergers at redshifts $z<1$ (van Dokkum et al. 1999). Hence the stars in cluster ellipticals form early, but the galaxies' final morphological forms may be more lately acquired.

[Francis] described a detailed investigation of a candidate galaxy cluster at $z=2.38$. Narrowband imaging of this cluster shows three bright Lyman- $\alpha$ sources, one of which is more than $50 h^{-1} \mathrm{kpc}$ in extent. Two of these sources have very red colours, and near-infrared pho- tometry shows that their spectral energy distributions are poorly-fit by dust-obscured star-forming regions, but well-fit by stellar populations with ages of $0.5 \mathrm{Gyr}$ and masses of a few times $10^{11} M_{\odot}$. A number of other objects with similar infrared colours are seen in the cluster, and have luminosity profiles more consistent with an $R^{1 / 4}$ law than an exponential disk. The cluster lies in front of three QSOs, and in all three sightlines there are neutral hydrogen absorption lines (including damped Lyman- $\alpha$ systems) at the cluster redshift. This suggests that the cluster contains a large amount of neutral hydrogen, perhaps more than $10^{12} M_{\odot}$, which may be in the form of large numbers of short-lived $10^{6}$ $M_{\odot}$ clouds.

[Madau] reviewed the constraints on the history of star formation and the mass density in stars that can be derived from the extragalactic background light (EBL). He notes that the logarithmic slope of the faint galaxy counts drops below 0.4 at wavelengths from the ultraviolet to the near-infrared at the limits achieved in the Hubble Deep Fields (Figure 6(left); Madau \& Pozzetti 2000). This implies that the EBL is converging, with the bulk of the light already observed (Figure 6(right)), and gives a lower limit to the EBL intensity over the range $0.2-2.2 \mu \mathrm{m}$ of $15 \mathrm{nW} \mathrm{m}^{-2} \mathrm{sr}^{-1}$. Combined with measurements of the far-infrared background light from $\mathrm{COBE}$, and making approximate corrections for unresolved sources and the wings of the galaxy light profiles, Madau estimates that the total EBL intensity is $55 \pm 20$ $\mathrm{nW} \mathrm{m} \mathrm{m}^{-2} \mathrm{sr}^{-1}$. Using stellar population models and assumptions about the stellar initial mass function, he finds that the EBL requires that the present-day mass density in processed gas and stars has a lower limit of $\Omega_{g+s} h^{2}>0.0013$, corresponding to $\left\langle M / L_{B}\right\rangle_{g+s}>3.5$. Plausible models for the star-formation history give $\Omega_{g+s}$ twice as large. Another constraint arising from the EBL relates to the possibility, raised by the MACHO gravitational lensing survey, that galaxies' dark halos may be composed of faint white dwarfs. Madau finds that in fact no more than $5 \%$ of the baryons can be in the form of stellar remnants without over-producing the EBL.

Cowie and Rowan-Robinson discussed what can be learnt about galaxy evolution by combining submillimetre observations with optical and infrared data. [Rowan-Robinson] presented models of the starformation history of the universe, based on results from imaging surveys of galaxies at infrared and submillimetre wavelengths. The European Large-Area ISO Survey (ELAIS; Rowan-Robinson et al. 1999) has employed the ISO satellite to image $4-12 \mathrm{deg}^{2}$ at 6.7 , 15,90 and $175 \mu \mathrm{m}$, reaching down to $3 \mathrm{mJy}$ at $15 \mu \mathrm{m}$. This survey is supported by optical and near-infrared imaging, $21 \mathrm{~cm}$ observations, and a SCUBA submillimetre survey of $0.02 \mathrm{deg}^{2}$ down to $8 \mathrm{mJy}$ at 850 $\mu \mathrm{m}$. Rowan-Robinson uses simple parametrised starformation models to fit the infrared and submillimetre galaxy counts (Rowan-Robinson 1999). He finds that 

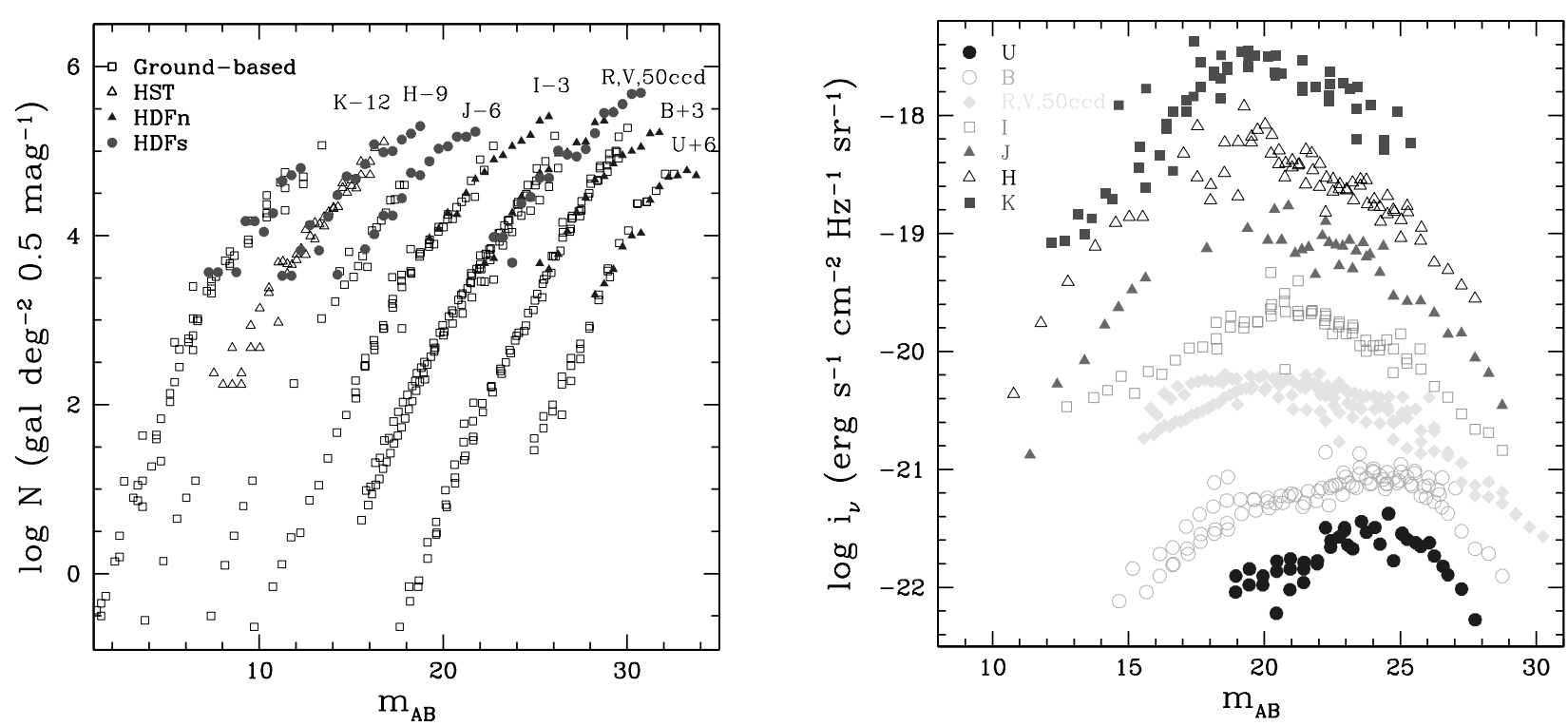

Figure 6-(left) The optical and near-infrared galaxy counts in various passbands as functions of AB magnitude. (right) The extragalactic background light per magnitude bin in each passband (arbitrarily scaled), showing the turnover and decreasing contributions at the faintest magnitudes (see Madau \& Pozzetti 2000).

the star-formation rate increases to $z \sim 1$ and then remains high out to at least $z \sim 5$. Within this framework, the submillimetre background intensity can be used to discriminate between cosmological models by comparing their predictions with the observed farinfrared and submillimetre background spectrum. A low-density $(\Omega \approx 0.3, \Lambda=0)$ model is preferred; a highdensity $(\Omega=1, \Lambda=0)$ model requires a spike in the starformation rate at $z>5$, while a flat $(\Omega \approx 0.3, \Lambda \approx 0.7)$ model requires truncation of star-formation at $z \geq 5$.

[Cowie] noted that the submillimetre background flux indicates that much of the energy produced by star formation and AGN is reprocessed by dust to farinfrared wavelengths. SCUBA observations at $850 \mu \mathrm{m}$ show that the background is due to ultra-luminous infrared galaxies at redshifts $z>1$. Submillimetre imaging has poor spatial resolution, but the fact that both submillimetre and centimetre flux are related to star-formation rate has meant that centimetre radio observations can be used to locate the submillimetre sources (Barger, Cowie \& Richards 1999). However, these objects turn out to be extremely faint in the optical and near-infrared, and inaccessible to spectroscopic follow-up. Nonetheless it is possible to estimate their redshifts from the shape of their spectral energy distribution at submillimetre and radio wavelengths. This procedure suggests they are at redshifts $z=1-3$; if so, the submillimetre sources are a major star-forming population at high redshift (Cowie \& Barger 1999).

[Calzetti] outlined models for the evolution of the star and gas contents of galaxies, giving special consideration to the effects of dust (Calzetti \& Heckman 1999). She estimates that the presence of dust suppresses the UV flux by a factor of $2.5-5$ at $z=1-3$, with a significant fraction of the radiation from star formation emerging in the far infrared. Star-formation histories with either low star-formation rates and low dust content at high redshift, or high star-formation rates and high dust content, are both compatible with the observed ultraviolet flux, but can differ by an order of magnitude in the numbers of stars produced at $z>3$. Both types of model predict that the far-infrared background is mostly produced by high-redshift sources, but the model with a constant star-formation rate for $1<z<4$ predicts that the dust will be hotter at high redshift.

\section{The Future}

The 'next generation' was the theme of the final session of the conference. [Glazebrook] discussed new observational techniques for redshift surveys. He described the application of the 'nod and shuffle' (also known as 'va et vient') and 'micro-slit' techniques, which together allow near-optimal sky-subtraction at faint magnitudes and a greatly increased multiplex gain. High-precision sky subtraction is achieved by simultaneously moving the electrons back and forth on the CCD (charge shuffling) in concert with moving the telescope back and forth on the sky (nodding). This exact differential beamswitching approach means that the object and the sky are observed through the same part of the slit (or the same fibre) and with the same pixels of the detector, but avoids the extra read noise imposed by simply nodding between exposures. It also offers more rapid time sampling. With near-perfect sky subtraction, long slits are not needed, so one can use small apertures (microslits) in the focal plane mask, and so observe many more objects simultaneously. Glazebrook described how these techniques (and the introduction of a volume-phase holographic grating) allowed the LDSS++ spectrograph on the $4 \mathrm{~m}$ AAT to measure redshifts in the Hubble Deep Field South to $R \approx 24$ in a 12 hour exposure (6 hours on-object). Further extensions to this technique 

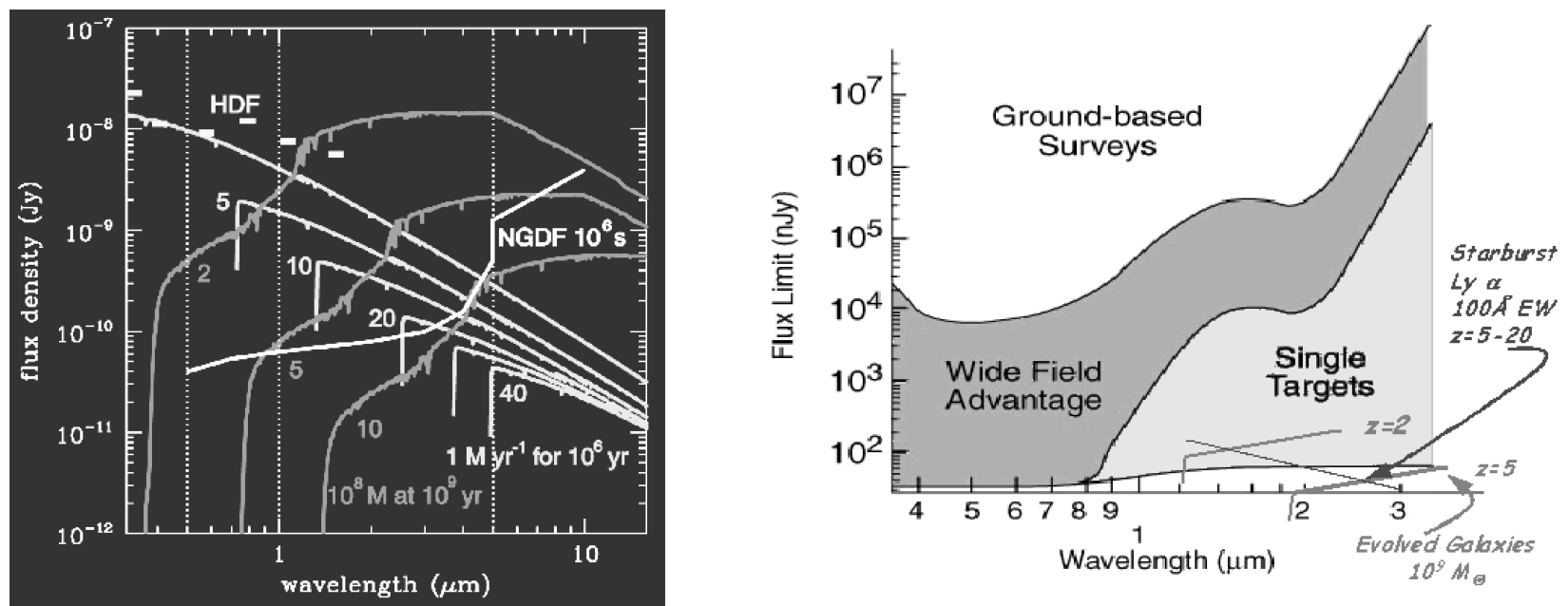

Figure 7-The capabilities of the Next Generation Space Telescope for imaging and spectroscopy: (left) The broadband flux limits, as a function of wavelength, reached by a proposed 'Next Generation Deep Field'. The two sets of spectral energy distributions shown correspond to a galaxy dominated by a young stellar population with a star-formation rate of $1 M_{\odot} \mathrm{yr}^{-1}$ (at $z=2,5,10,20$ and 40 ), and a $10^{8} M_{\odot}$ galaxy with an old (1 Gyr) stellar population (at $z=2,5$ and 10). (right) The spectroscopic flux limits, as a function of wavelength, achievable at a resolving power of 1000 by ground-based telescopes (with and without wide-field capability) and by NGST. The spectra of galaxies with young and old stellar populations are indicated schematically.

include the use of blocking filters to isolate small spectral ranges and so allow more spectra to be crammed onto the detector. Alternatively, one can use a 'pseudo-slitless' mode in which the object spectra are allowed to overlap (as in objective prism spectroscopy, but with a mask). The ambiguity of which line belongs to which object can be resolved by obtaining a second set of spectra with the grating rotated through $180^{\circ}$. Glazebrook outlined some case studies showing how these techniques could significantly improve the grasp (in terms of both depth and sample size) of the next generation of redshift surveys.

Large, deep redshift surveys, and particularly surveys of relatively rare objects, require deep, widefield imaging to select the target samples. [Sutherland] described the plans for a next-generation survey telescope, the $4 \mathrm{~m}$ Visible-Infrared Survey Telescope for Astronomy (VISTA). In the visible, VISTA will have a $1.5^{\circ} \times 1.5^{\circ}$ field of view, provided by a mosaic of 50 $2048 \times 4096$ CCDs; in the near-infrared it will be able to cover $1.0^{\circ} \times 1.0^{\circ}$ with four pointings of its nine $2048 \times$ 2048 IR arrays. The observational strengths will be large surveys (e.g. weak lensing, photometric redshifts), searches for rare objects (e.g. very-high-redshift QSOs and galaxies, brown dwarfs) and studies of variable objects (supernovae, microlensing, Kuiper belt objects). VISTA will be located in Chile, possibly on Cerro Pachon, and the current target is to achieve first light in late 2003.

The Next Generation Space Telescope (NGST) will be an extraordinarily potent probe of the origin of galaxies and cosmology. [Christian] reviewed the scientific goals and likely configuration of NGST. The baseline plan calls for an $8 \mathrm{~m}$ telescope with wavelength coverage from $0.6-10+\mu \mathrm{m}$ to be launched in 2008 . The Design Reference Mission, which specifies the core science goals of NGST, includes deep imaging and spectroscopic surveys of galaxy formation and evolution. A deep imaging survey with NGST could in principle detect a $10^{8} M_{\odot}$ galaxy with an old stellar population out to $z>5$, and a galaxy forming stars at the rate of $1 M_{\odot} \mathrm{yr}^{-1}$ to $z>10$ (see Figure $7($ left)). A spectroscopic survey at a resolving power of 1000 could measure the star-formation rate in normal galaxies at $z \approx$ 2 and in starburst galaxies at $z>5$ (see Figure 7(right)). These capabilities will allow the direct observation of the processes of star formation in the very early universe.

\section{Acknowledgments}

I thank Luigi Guzzo for macros used in producing Figure 1, Scott Croom for some of the data used in producing Figure 3, John Peacock for Figure 4, the SDSS team for Figure 5, Piero Madau for Figure 6, and Carol Christian and Simon Lilly for Figure 7.

\section{References}

Barger, A. J., Cowie, L. L., \& Richards, E. A., 1999, in Photometric Redshifts and High-Redshift Galaxies, ASP Conf. Ser., ed. R. Weymann et al. (San Francisco: ASP), p. 279

Baugh, C. M., \& Efstathiou, G. 1993, MNRAS, 265, 145

Boyle, B. J., Croom, S. M., Smith, R. J., Shanks, T., Miller, L., \& Loaring, N. 1999, in Looking Deep in the Southern Sky, ed. R. Morganti \& W. J. Couch (Berlin: Springer), p. 16

Calzetti, D. 1997, AJ, 113, 162

Calzetti, D., \& Heckman, T. M. 1999, ApJ, 519, 27

Chester, T., Jarrett, T., Schneider, S., Skrutskie, M., \& Huchra, J. 1998, BAAS, 192, 5511

Colless, M. M. 1998, in Wide Field Surveys in Cosmology, 14th IAP Colloquium, ed. S. Colombi, Y. Mellier \& B. Raban, 14th IAP Colloquium (Editions Frontières: Paris), p. 77

Colless, M. M. 1999, Phil. Trans. R. Soc. Lond. A, 357, 105 
Cowie, L. L., \& Barger, A. J. 1999, in The High-Redshift Universe: Galaxy Formation and Evolution at High Redshift, ASP Conf. Ser., ed. A. J. Bunker \& W. J. M. van Breughel (San Francisco: ASP), in press

Davis M., \& Faber S. M. 1998, in Wide Field Surveys in Cosmology, ed. S. Colombi, Y. Mellier \& B. Raban, 14th IAP Colloquium, (Editions Frontières: Paris), p. 333

Ellis, R. S., Colless, M. M., Broadhurst, T., Heyl, J., \& Glazebrook, K. 1996, MNRAS, 280, 235

Fan, X., et al. 1999, AJ, 118, 1

Folkes, S., et al. 1999, MNRAS, 308, 459

Giavalisco, M., Steidel, C. C., Adelberger, K. L., Dickinson, M., Pettini, M., \& Kellogg, M. 1998, ApJ, 503, 543

Guzzo, L., et al. 1999, ESO Messenger, 95, 27

Kelson, D. D., Illingworth, G. D., van Dokkum, P. G., \& Franx, M. 2000, ApJ, 531, 137

Kilborn, K., Webster, R. L., \& Staveley-Smith, L. 1999, PASA, 16,8

Koo, D. C. 1998, in Wide Field Surveys in Cosmology, 14th IAP Colloquium, ed. S. Colombi, Y. Mellier \& B. Raban, 14th IAP Colloquium (Editions Frontières: Paris), p. 161

LeFevre, O., et al. 1999, in Observational Cosmology: The Development of Galaxy Systems, ASP Conf. Ser. 176, ed. G. Giuricin, M. Mezzetti \& P. Salucci (San Francisco: ASP), p. 250

Loveday, J., Peterson, B. A., Efstathiou, G., \& Maddox, S. J. 1992, ApJ, 390, 338

Loveday, J., \& Pier, J. R. 1998, in Wide Field Surveys in Cosmology, 14th IAP Colloquium, ed. S. Colombi, Y. Mellier \& B. Raban (Editions Frontières: Paris), p. 317

Madau, P., \& Pozzetti, L. 2000, MNRAS, 312, 9

Maddox, S. J., Sutherland, W. J., Efstathiou, G., Loveday, J., \& Peterson, B. A. 1990, MNRAS, 247, 1P
Mamon, G. A. 1999, in Cosmic Flows: Towards an Understanding of the Large-Scale Structure of the Universe, ASP Conf. Ser., ed. S. Courteau, M. Strauss \& J. Willick (San Francisco: ASP), p. 103

Margon, B. 1999, Phil. Trans. Roy. Soc. Lond. A, 357, 93

Mo, H. J., Mao, S., \& White, S. D. M. 1999, MNRAS, 304, 175

Mould, J. R., et al. 2000, ApJ, 529, 786

Rowan-Robinson, M., et al. 1999, in The Universe as seen by ISO, ESA Special Publ. Ser. SP-427, ed. P. Cox \& M.F. Kessler, p. 1011

Rowan-Robinson, M. 2000, MNRAS, 316, 885

Sadler, E. M., McIntyre, V. J., Jackson, C. A., \& Cannon, R. D. 1999, PASA, 16, 247

Schmoldt, I. M., et al. 1999a, MNRAS, 304, 893

Schmoldt, I. M., et al. 1999b, AJ, 118, 1146

Schuecker, P., et al. 1998, in 19th Texas Symposium on Relativistic Astrophysics and Cosmology, ed. J. Paul, T. Montmerle \& E. Aubourg (Saclay: CEA), p. 546

Strauss, M. A., et al. 1999, ApJ, 522, L61

Staveley-Smith, L., Webster, R. L., Banks, G., Kilborn, K., Koribalski, B., \& Putman, M. 1999, in Looking Deep in the Southern Sky, ed. R. Morganti \& W. J. Couch (Berlin: Springer), p. 132

Steidel, C. C., Adelberger, K. L., Giavalisco, M., Dickinson, M., \& Pettini, M. 1999, ApJ, 519, 1

Sutherland, W., et al. 1999, MNRAS, 308, 289

Tadros, H., et al. 1999, MNRAS, 305, 527

Tadros, H., Efstathiou, G., \& Dalton, G. 1998, MNRAS, 296, 995

van Dokkum, P. G., Franx, M., Fabricant, D., Kelson, D. D., \& Illingworth, G. D. 1999, ApJ, 520, 95L

Vettolani, G., et al. 1997, A\&A, 325, 954

Vettolani, G., et al. 1998, A\&AS, 130, 323

Zucca, E., et al. 1997, A\&A, 326, 477 